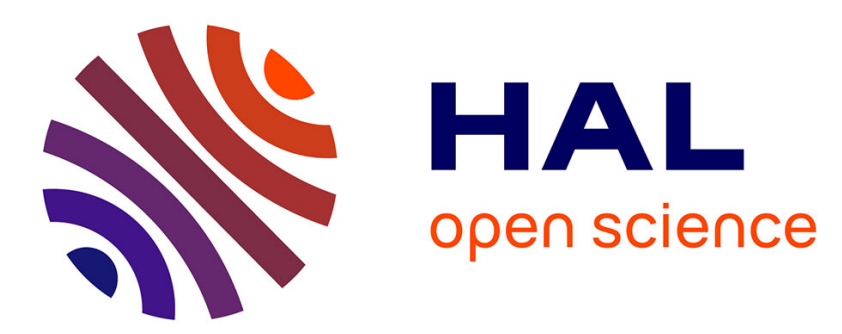

\title{
L'inoculation des Eucalyptus introduits en Asie avec des champignons ectomycorhiziens australiens en vue d'augmenter la productivité des plantations.
}

\author{
B. Dell, N. Malajczuk
}

\section{- To cite this version:}

B. Dell, N. Malajczuk. L'inoculation des Eucalyptus introduits en Asie avec des champignons ectomycorhiziens australiens en vue d'augmenter la productivité des plantations.. Revue forestière française, 1997, 49 (sp), pp.174-184. 10.4267/2042/5667 . hal-03444114

HAL Id: hal-03444114

https://hal.science/hal-03444114

Submitted on 23 Nov 2021

HAL is a multi-disciplinary open access archive for the deposit and dissemination of scientific research documents, whether they are published or not. The documents may come from teaching and research institutions in France or abroad, or from public or private research centers.
L'archive ouverte pluridisciplinaire HAL, est destinée au dépôt et à la diffusion de documents scientifiques de niveau recherche, publiés ou non, émanant des établissements d'enseignement et de recherche français ou étrangers, des laboratoires publics ou privés. 


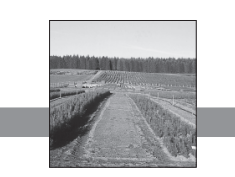

\title{
L'INOCULATION DES EUCALYPTUS INTRODUITS EN ASIE AVEC DES CHAMPIGNONS ECTOMYCORHIZIENS AUSTRALIENS EN VUE D'AUGMENTER LA PRODUCTIVITÉ DES PLANTATIONS
}

\author{
B. DELL - N. MALAJCZUK
}

Dans beaucoup de régions de Chine et des Philippines, des sols dégradés qui étaient auparavant colonisés par des forêts de Fagacées, de Pinacées ou de Diptérocarpacées, sont reboisés à l'aide d'Eucalyptus à croissance rapide. Ces plantations permettent de stabiliser les sols et assurent une production forestière. Néanmoins, beaucoup de ces plantations ont une faible productivité. Cet état de fait peut être attribué à la faible disponibilité en éléments minéraux du sol (Dell et Malajczuk, 1994) et à l'absence de champignons ectomycorhiziens adaptés à l'Eucalyptus (Malajczuk, Brundrett et Dell, 1995). En Australie, il y a une grande diversité de champignons ectomycorhiziens associés à l'Eucalyptus dans son aire naturelle (Castellano et Bougher, 1994 ; Bougher, 1995). Nos propres observations montrent que très peu de ces champignons ont été introduits dans les plantations commerciales d'Eucalyptus en Asie. De plus, là où l'Eucalyptus a été introduit en dehors de son aire naturelle, il s'associe avec un nombre limité de champignons ectomycorhiziens. Généralement, dans ces conditions, il contracte des associations avec des champignons qui ont un large spectre d'hôtes comme les espèces des genres Pisolithus et Scleroderma. Ces espèces n'ont qu'une faible affinité avec l'Eucalyptus (Malajczuk et al., 1990).

Dans ces conditions, nous pouvons supposer que l'Eucalyptus inoculé avec des souches compatibles de champignons ectomycorhiziens devrait avoir un meilleur comportement que l'Eucalyptus s'associant avec les souches indigènes locales n'ayant que peu d'affinité avec lui. Le Centre australien pour la Recherche internationale en Agriculture a soutenu des recherches en collaboration entre l'Australie et l'Asie du Sud-Est dans l'optique d'évaluer les possibilités de développement de l'inoculation contrôlée de l'Eucalyptus en pépinière et après plantation. Une partie de ce programme implique la sélection de souches ectomycorhiziennes associées à l'Eucalyptus sur sols lessivés acides en climat sub-tropical. Dans cet article, nous présentons les résultats de croissance, dans les provinces du Yunnan en Chine et de Mindanao aux Philippines, de semis inoculés en pépinière. Ces exemples illustrent les possibilités d'inoculation dans des pépinières forestières classiques ou plus sophistiquées. 


\section{MATÉRIELS ET MÉTHODES}

\section{Sites expérimentaux}

\section{- Chine}

Les essais de terrain ont été installés près de la ville de Kunming à Jin Dian et Tai Ping sur le plateau du Yunnan, à une altitude moyenne de $1900 \mathrm{~m}$. Le climat de mousson est caractérisé par une saison humide qui va de juin à septembre. Les sols sont acides, de couleur jaune à rouge, avec une faible disponibilité en azote et en phosphore et une faible teneur en matière organique (tableau I, cidessous). Ils ont une teneur élevée en manganèse extractible et sont déficients en bore $(<0,2 \mathrm{mg}$ de B soluble dans l'eau g-1 de sol ; Dell et Malajczuk, 1994). Avant les plantations d'Eucalyptus, la végétation était dominée par des conifères chétifs, principalement Pinus yunnanensis et Pinus armandii.

Tableau I Propriétés chimiques des horizons de surface $(0-10 \mathrm{~cm})$ des quatre sites expérimentaux

\begin{tabular}{|c|c|c|c|c|c|}
\hline Site & $\begin{array}{c}\mathrm{pH} \\
\text { 1:5 sol:eau }\end{array}$ & $\begin{array}{c}\text { P total (\%) } \\
\text { (Murphy et Riley, } \\
\text { 1962) }\end{array}$ & $\begin{array}{c}\mathrm{P} \text { Bray (g/kg) } \\
\text { (Bray et Kurtz, } \\
\text { 1945) }\end{array}$ & $\begin{array}{c}\mathrm{N} \text { total (\%) } \\
\text { (Murphy et Riley, } \\
\text { 1962) }\end{array}$ & $\begin{array}{c}\text { Carbone } \\
\text { organique (\%) } \\
\text { (Walkley, 1947) }\end{array}$ \\
\hline Jin Dian ...... & 4,2 & 0,038 & 0,71 & 0,09 & 1,67 \\
Tai Ping ...... & 4,5 & 0,015 & 0,31 & 0,03 & 0,45 \\
Bislig ....... & 5,9 & - & 0,21 & 0,06 & 1,14 \\
Malaybalay ... & 4,3 & - & 0,12 & 0,37 & 4,35 \\
\hline
\end{tabular}

\section{- Philippines}

Les essais de terrain ont été installés dans deux sites de l'île de Mindanao : Bislig à Surigao Sur et près de Malaybalay à Manolo Fortich dans la province du Bukidnon. Le site de Bislig était auparavant une plantation sur limons de Paraserianthes falcata qui a été entièrement exploitée. Originellement le site était occupé par une forêt tropicale humide de basse altitude dominée par les diptérocarpacées et gérée par la société Paper Industries Corporation des Philippines (PICOP). Le site de Malaybalay était originellement une forêt tropicale humide de montagne. Avant la plantation d'Eucalyptus par la société Bukidnon Forest Industries, le site était occupé par des graminées, principalement du Cogon (Imperata cylindrica) et du Bagokbok (Themeda triandra). La saison sèche est courte et va de décembre à avril. Le sol, de texture limono-sableuse, est issu de matériel volcanique.

\section{Inoculum fongique}

L'inoculum des différents champignons ectomycorhiziens (tableau II, p. 177) a été produit par les méthodes suivantes (Brundrett et al., 1996) :

- Spores : les spores sont obtenues soit à partir de carpophores séchés puis conservés à température ambiante en Australie, soit à partir de carpophores frais mis à macérer dans de l'eau stérile et conservés à $4{ }^{\circ} \mathrm{C}$ jusqu'à utilisation.

- Inoculum solide : il a été produit suivant la méthode décrite par Marx et Kenney (1982). Un kilogramme de substrat, contenant de la vermiculite, de la tourbe et des copeaux de bois de pin (11:1:2 en volume) et saturé par une solution de Melin Norkran modifiée (Marx, 1977), est autoclavé dans des sacs de cellophane puis inoculé par une souche fongique et mis à incuber à $20-30{ }^{\circ} \mathrm{C}$ pendant 30 à 60 jours. 
- Mycélium produit in vitro : le mycélium est produit sur un milieu de Melin Norkran modifié solidifié par de l'agar dans des flacons de $500 \mathrm{ml}$ en polycarbonate.

- Alginate : le mycélium est produit dans des billes d'alginate (Kuek, Tommerup et Malajczuk, 1992).

\section{L'inoculation des semis}

- Chine

Pour l'essai de Jin Dian, l'inoculum (tableau II, p. 177) a été introduit dans le sol de pépinière en petits sacs en plastique d'environ $1 \mathrm{~kg}$. Des graines ou de jeunes semis de 1 à $2 \mathrm{~cm}$ de hauteur d'Eucalyptus globulus (origine Geevston, Tasmanie) produits sur substrat désinfecté, ont été semés ou repiqués au-dessus de l'inoculum. Le sol de pépinière, constitué d'un mélange 3 pour 1 en volume de sol tamisé et d'humus également tamisé, a été au préalable désinfecté par saturation au formol. Une solution à $0,5 \%$ a été appliquée pendant 10 jours sous une feuille de plastique. Après enlèvement du plastique, le formol a pu s'évaporer pendant un mois.

À Tai Ping, des semis axéniques ont été déposés sur un lit de mycélium développé sur agar (tableau II, p. 177) dans des tubes de polycarbonate. Après trois semaines, ils ont été transplantés sur un substrat tourbe vermiculite autoclavé (1:1 en volume) dans des plaques en plastique à 64 alvéoles. Les semis témoins ont été produits dans les mêmes conditions, mais sans apport d'inoculum fongique. Les plantules ont été arrosées une fois par semaine par une solution nutritive complète $\left(3 \mathrm{ml} \mathrm{Wuxol}{ }^{\mathrm{TM}}\right)$. Après deux à trois mois de culture, les jeunes plants ont été transplantés sur le terrain (juillet 1992).

\section{- Philippines}

Des semis axéniques d'Eucalyptus urophylla (origine Mt Egon, Indonésie) ont été déposés sur un lit de mycélium développé sur agar (tableau II, p. 177) à Perth et après trois semaines transportés à Mindanao. À Malaybalay, les semis ont été transplantés dans un compost de bagasse de canne à sucre $(\mathrm{pH} 5,4)$ dans des tubes à rainures pour racines dans la pépinière de la société Bukidnon Forest Industries. Le régime de fertilisation a été le même qu'à Tai Ping. Après quatre mois, les jeunes plants ont été introduits sur le terrain (juin 1993).

À Bislig, les semis ont été transférés dans un sable de rivière $(\mathrm{pH} 6,9)$ contenu dans des récipients de $70 \mathrm{~cm}^{3}$. Chaque conteneur a reçu $2 \mathrm{~g}$ d'un engrais complet (14 $\mathrm{N}: 14 \mathrm{P}_{2} \mathrm{O}_{5}: 14 \mathrm{~K}_{2} \mathrm{O}$ ). Ils ont été transplantés sur le terrain en octobre 1993 après deux mois de croissance dans la pépinière de PICOP.

\section{Les essais de terrain}

À Jin Dian et Malaybalay, les essais ont été implantés en blocs complets aléatoires. Chaque bloc a été répété quatre fois. Les placeaux unitaires étaient constitués d'un rang de 10 à 12 arbres avec espacement d'un mètre entre les rangs. Dans les deux autres sites, les traitements élémentaires étaient constitués d'un rang de 10 plants disposés en trois blocs (Brundrett et al., 1996). Avant plantation, le sol a été préparé par des trous individuels (Jin Dian, Bislig), par tranchée (Tai Ping) ou par décapage au bulldozer (Malaybalay). Une fertilisation de fond a été appliquée à la plantation sur une bande de 30 à $50 \mathrm{~cm}$ de part et d'autre du tronc, à raison de 100 à $130 \mathrm{~g}$ de NPK par arbre, 40 à $60 \mathrm{~g}$ d'urée et 25 à $40 \mathrm{~g}$ d'un mélange d'oligo-éléments $(1,5 \% \mathrm{Cu}, 0,26 \% \mathrm{Zn}, 3,9 \% \mathrm{Mn}$, $6,0 \% \mathrm{Mg}, 5,7 \% \mathrm{Fe}, 0,19 \% \mathrm{Mo}, 0,13 \% \mathrm{~B}$ et 0,03\% Co) par arbre. Aux Philippines, chaque arbre a reçu à la plantation $100 \mathrm{~g}$ d'un engrais complet (keiserite) et, après deux mois, $100 \mathrm{~g}$ d'urée. Les 
niveaux de fertilisation ont été choisis en fonction des analyses de sol et en fonction des résultats d'essais de fertilisation. Le niveau de fertilisation choisi pour le phosphore a été de $20-30 \%$ de la dose requise, dans ces sols à fort pouvoir fixateur de phosphore, pour la croissance optimale d'arbres non inoculés.

\section{Les mesures}

Le diamètre de la tige et la hauteur ont été mesurés à des intervalles de 4 à 36 mois après la plantation. Les effets des traitements ont été analysés par le programme statistique GENSTAT (Rothamsted Experimental Station).

Tableau II

Espèces fongiques et type d'inoculum utilisé dans les quatre sites

TP = Tai Ping, JD = Jin Dian, $\mathrm{P}=$ Philippines (Malaybalay et Bislig)

\begin{tabular}{|c|c|c|c|c|}
\hline Champignon & Code & Origine & Forme d'inoculum & Site \\
\hline Cortinarius lavendulensis & E4062 & $\begin{array}{l}\text { Ouest } \\
\text { de l'Australie }\end{array}$ & mycélium in vitro & TP \\
\hline Descomyces albellus & $\mathrm{H} 584$ & $\begin{array}{l}\text { Ouest } \\
\text { de l'Australie }\end{array}$ & $\begin{array}{l}\text { spores en suspension } \\
\text { dans l'eau }\end{array}$ & $J D$ \\
\hline Descolea maculata. & E4071 & $\begin{array}{l}\text { Ouest } \\
\text { de l'Australie }\end{array}$ & mycélium in vitro & TP \\
\hline Hebeloma westraliense & E4070 & $\begin{array}{l}\text { Ouest } \\
\text { de l'Australie }\end{array}$ & mycélium in vitro & TP \\
\hline Hydnangium sublamellatum & Hyd 1 & $\begin{array}{l}\text { Ouest } \\
\text { de l'Australie }\end{array}$ & spores séchées & JD \\
\hline Hydnangium sublamellatum & Hyd 2 & $\begin{array}{l}\text { Ouest } \\
\text { de l'Australie }\end{array}$ & $\begin{array}{l}\text { spores en suspension } \\
\text { dans l'eau }\end{array}$ & JD \\
\hline Laccaria laccata ........ & E1045 & Victoria & billes d'alginate & JD \\
\hline Laccaria laccata & E4100 & Queensland & mycélium in vitro & TP, $\mathrm{P}$ \\
\hline Pisolithus sp. & $\mathrm{H} 445$ & $\begin{array}{l}\text { Ouest } \\
\text { de l'Australie }\end{array}$ & $\begin{array}{l}\text { tourbe/vermiculite/ } \\
\text { écorce }\end{array}$ & JD, TP \\
\hline Pisolithus sp. . ........... & $\mathrm{H} 636$ & $\begin{array}{l}\text { Ouest } \\
\text { de l'Australie }\end{array}$ & spores séchées & JD \\
\hline Pisolithus sp. & $\mathrm{H} 4111$ & Queensland & mycélium in vitro & TP \\
\hline Setchelliogaster sp. . & H51023 & Tasmanie & mycélium in vitro & TP \\
\hline Scleroderma sp. & H587 & $\begin{array}{l}\text { Ouest } \\
\text { de l'Australie }\end{array}$ & $\begin{array}{l}\text { spores en suspension } \\
\text { dans l'eau }\end{array}$ & $J D$ \\
\hline Scleroderma sp. ..... & H5109 & Tasmanie & mycélium in vitro & TP \\
\hline Scleroderma sp. & H5556 & $\begin{array}{l}\text { Ouest } \\
\text { de l'Australie }\end{array}$ & mycélium in vitro & $\mathrm{P}$ \\
\hline Scleroderma sp. .... & H6266 & Philippines & mycélium in vitro & $P$ \\
\hline Thaxterogaster sp. ......... & $\mathrm{H} 5141$ & Tasmanie & mycélium in vitro & TP \\
\hline Tylopilus sp. & E4240 & Queensland & mycélium in vitro & TP \\
\hline
\end{tabular}




\section{RÉSULTATS}

Dans tous les essais, l'inoculation par des champignons ectomycorhiziens australiens a eu un effet sur la croissance par rapport aux traitements témoins non inoculés. Les différences sont aussi sensibles entre les traitements fongiques, certains pouvant être dépressifs.

À Jin Dian, les différences entre les traitements ont commencé à apparaître au bout de neuf mois (probabilité d'être non significatif $<0,10$ ) et sont devenues plus significatives à 16 mois (probabilité $<0,05)$. Les meilleurs résultats ont été les traitements avec Pisolithus et Hydnangium. À 36 mois, les arbres du traitement Hydnangium (Hyd2) avaient une surface terrière double de celle des témoins (figure 1, ci-dessous).

À Tai Ping, certains traitements (Hebeloma, Setchelliogaster, Pisolithus et Laccaria) avaient déjà un effet sur le diamètre des tiges trois ou quatre mois après la transplantation (figure 2, ci-dessous). Cet essai a été analysé plus en détail par Dell et Malajczuk (1994). Aux Philippines, l'inoculation par une souche de Scleroderma à Bislig et par plusieurs souches de Scleroderma et une souche de Laccaria laccata à Malaybalay stimule fortement la croissance de l'Eucalyptus deux ans après la plantation (figures 3 et 4 , p. 179).

Figure 1 EFFET DES TRAITEMENTS FONGIQUES SUR LA SURFACE TERRIÈRE DES TIGES D'EUCALYPTUS GLOBULUS, 3 ANS ET 9 MOIS APRĖS LA PLANTATION À JIN DIAN

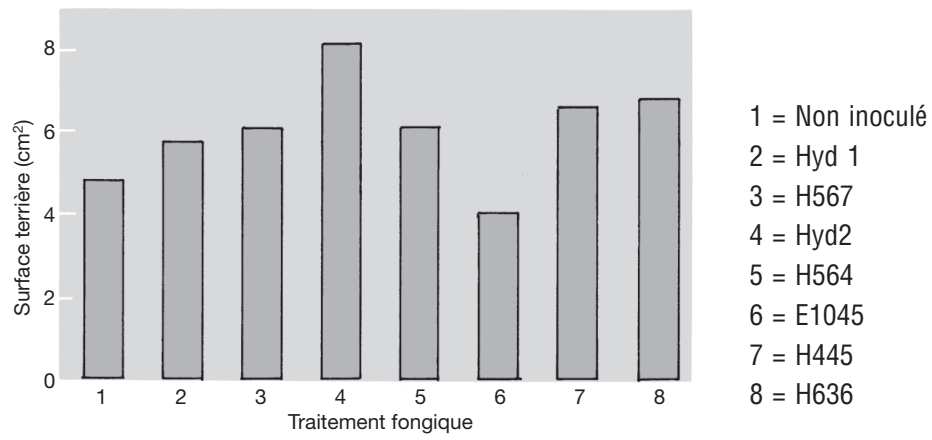

Figure 2 EFFET DES TRAITEMENTS FONGIQUES SUR LE DIAMÈTRE DES TIGES D'EUCALYPTUS GLOBULUS 4 MOIS APRĖS LA PLANTATION À TAI PING

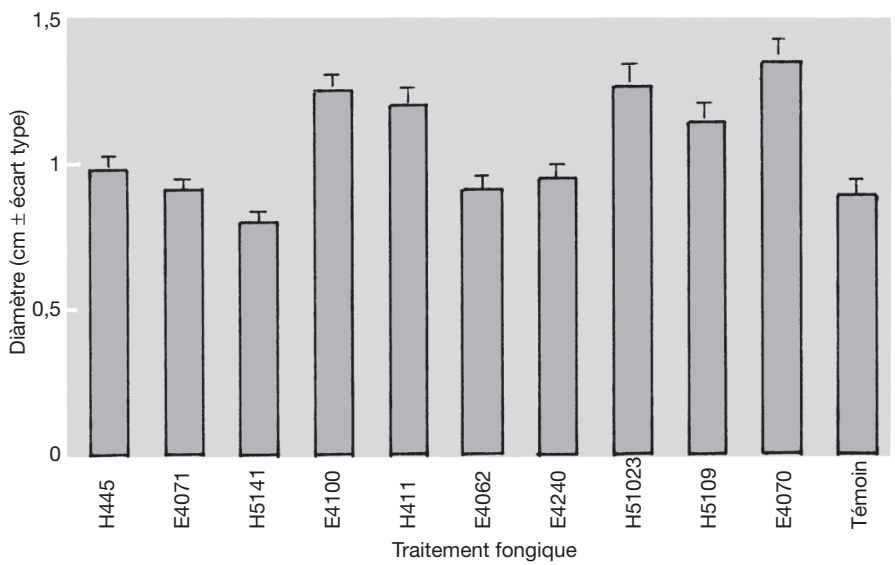

Les écarts types des moyennes individuelles sont représentés par des barres. 

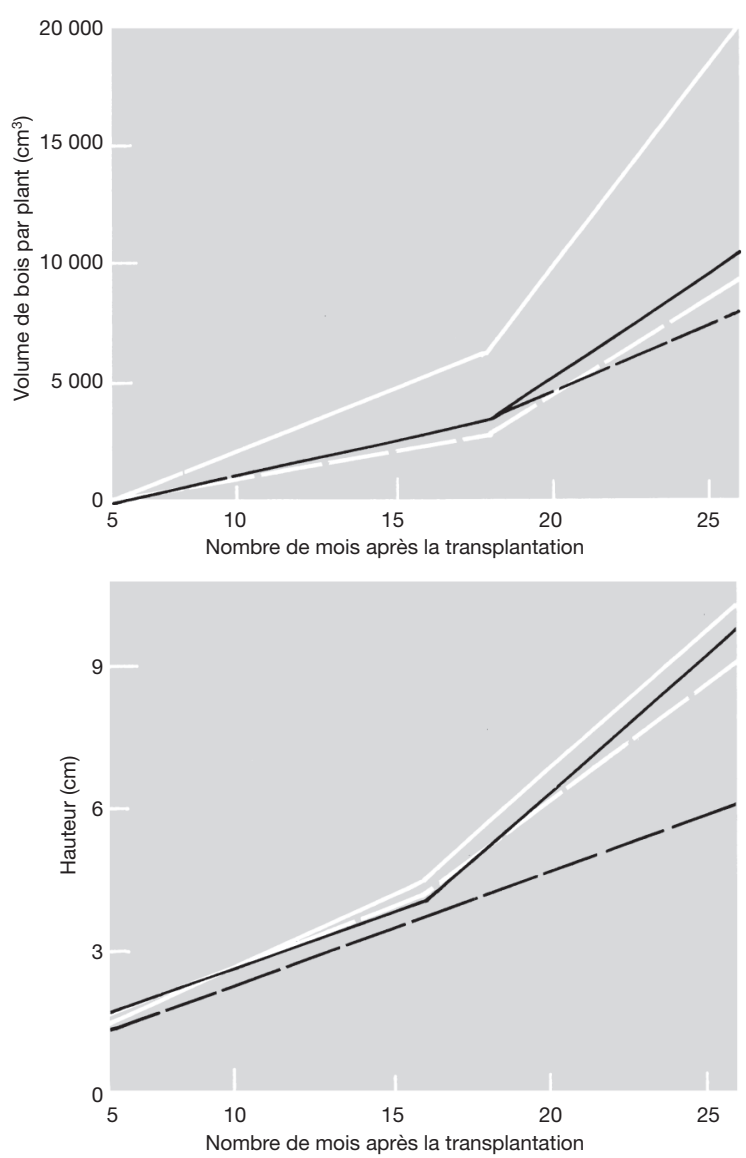

Figure 3

EFFET DE L'INOCULATION

AVEC SCLERODERMA ET LACCARIA

sur la production en volume d'Eucalyptus urophylla sur deux ans dans le site de Bislig, Mindanao (climat tropical humide de basse altitude)

La plus petite différence significative a été calculée pour $\mathrm{P}<0,01$.

- - Témoin

$=\mathrm{H} 6266$

E4100

H5556

Figure 4

EFFET DE L'INOCULATION

AVEC SCLERODERMA ET LACCARIA

sur la croissance en hauteur d'Eucalyptus urophylla sur deux ans dans le site de Malaybalay, Mindanao (climat tropical humide d'altitude, site secondairement colonisé par des graminées après disparition des diptérocarpacées)

La plus petite différence significative a été calculée pour $\mathrm{P}<0,05$.

- - Témoin

H6266

E4100

$\mathrm{H} 5556$

\section{DISCUSSION}

Ces essais en Chine et aux Philippines illustrent les bénéfices potentiels que l'on peut attendre en plantation de l'inoculation contrôlée de l'Eucalyptus. Les réponses précoces que nous avons obtenues sont essentielles pour l'établissement des plantations dans ces conditions de sites très perturbés où la compétition de la végétation adventice a un effet très négatif. Bien que non présentés ici, d'autres résultats montrent que, pour un même genre ou une même espèce, les souches australiennes de champignons ectomycorhiziens sont plus efficaces que les souches locales de Chine ou des Philippines. L'approche que nous avons adoptée pour la sélection de souches de champignons ectomycorhiziens adaptées aux plantations d'Eucalyptus en Asie a été détaillée par ailleurs (Malajczuk et al., 1995 ; Brundrett et al., 1996). Elle implique une sélection préliminaire de compatibilité avec l'Eucalyptus, une deuxième sélection basée sur la capacité des souches à stimuler la croissance de l'Eucalyptus sur sol à faible disponibilité en phosphore (Bruges, Malajczuk et Grove, 1993), et enfin sur une dernière sélection basée sur l'efficacité réelle des souches en plantation en Asie (Malajczuk et al., 1994, 1995). Les champignons sélectionnés doivent être capables de coloniser les racines d'Eucalyptus en condition de pépinière, survivre au choc de la crise de transplantation, persister, puis coloniser les nouvelles racines formées et enfin améliorer la survie et la croissance de l'Eucalyptus. 
Les possibilités d'application en vue de l'amélioration de la production forestière

Photo 5

Réponse six mois après plantation d'Eucalyptus grandis inoculé en pépinière avec un champignon australien. Le placeau inoculé est sur la gauche. Province de Guandong en Chine.

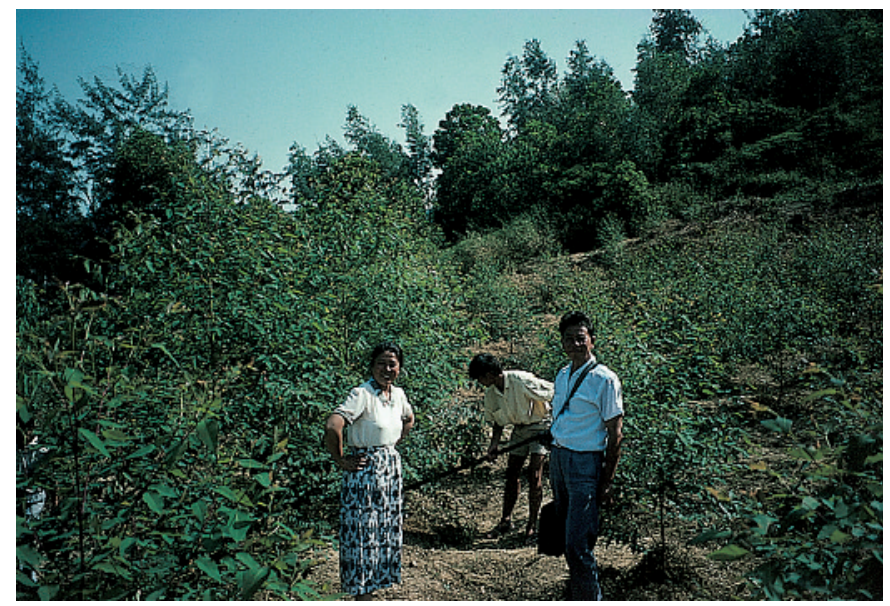

Photo 6 Site caractéristique de Bukidnon, Mindanao, Philippines (cf. figure 4, p. 179). La forêt tropicale humide d'altitude a été remplacée par une formation à graminées.

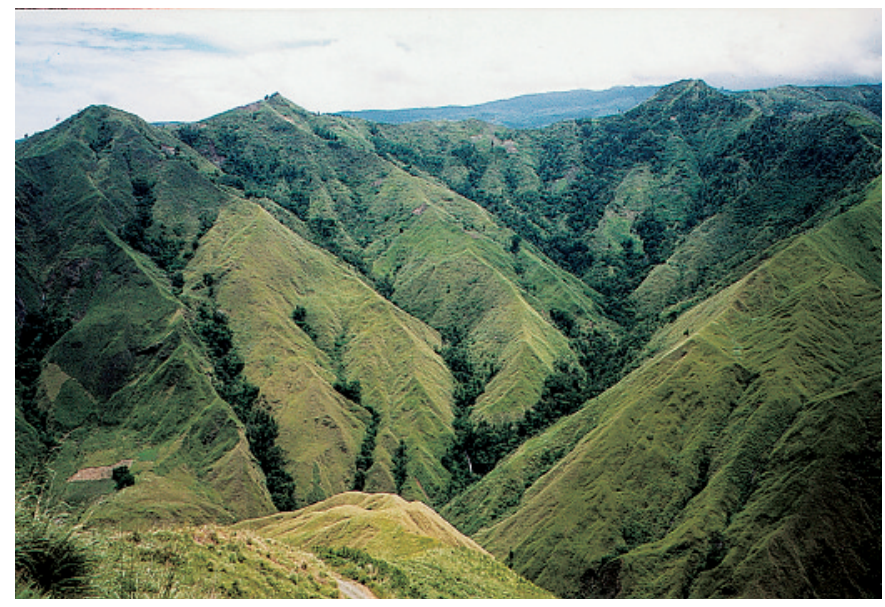

Photo 7 Site de Bislig, Mindanao, Philippines (cf. figure 3, p. 179). Forêt tropicale humide mixte de basse altitude préalablement plantée avec Paraserianthes falcata.

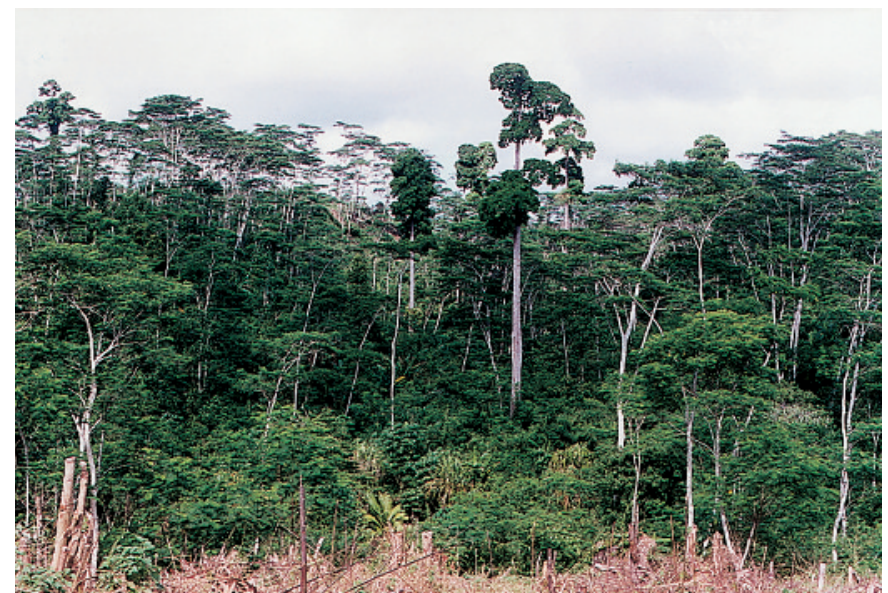


Les résultats de ces essais préliminaires démontrent d'autre part qu'il n'est pas possible de prédire les effets au "champ" des souches sélectionnées à partir d'essais en pots. Néanmoins nous essayons de construire des modèles prédictifs basés sur les effets des souches sur la croissance ainsi que sur leurs optima climatiques et édaphiques. Nous nous sommes également rendu compte qu'il était nécessaire d'éduquer le personnel des pépinières de façon à optimiser les paramètres de production (drainage, composition du substrat de culture, désinfection, contrôle des maladies des racines, régime de fertilisation) en vue de favoriser au maximum avant plantation le développement des ectomycorhizes sur des plants vigoureux et sains.

La forme de l'inoculum constitue une des clés de la réussite du développement de la mycorhization contrôlée. Lorsque la croissance est très rapide et que les semis ne restent que très peu de temps en pépinière comme à Bislig (deux mois), l'inoculation par spores est inadaptée. Les spores ne bénéficient en effet pas d'un temps suffisamment long pour germer. Actuellement, une formulation des spores en granulé est recommandée aux Philippines en foresterie (de La Cruz et al., 1990). Le développement de méthodes permettant la détermination des premiers stades de formation des mycorhizes après la germination des spores en pépinière est essentiel. Enfin, le système de culture axénique utilisé pour l'inoculation des jeunes semis et modifié pour la micropropagation végétative de l'Eucalyptus (Malajczuk et Hartney, 1986) a été utilisé avec succès dans des essais de terrain pour la micropropagation d'Eucalyptus urophylla et d'hybrides dans le Sud de la Chine. Nous pensons que cette méthode pourrait être utilisée plus largement, en particulier si les plantations clonales d'Eucalyptus se développent en Asie.

Ce travail de recherche en Asie est le développement d'études plus anciennes qui ont montré qu'il était possible d'obtenir en conditions tropicales humides des gains de croissance dans les plantations d'Eucalyptus après inoculation en pépinière (Garbaye et al., 1988 ; de La Cruz et al., 1988). Pour les plantations d'Eucalyptus en conditions tempérées, des travaux menés dans le Sud-Ouest de l'Australie ont montré qu'il était possible d'obtenir une amélioration de la croissance en plantation par inoculation préalable en pépinière (Grove et al., 1991; Grove et Le Tacon, 1993 ; Thompson et al., 1994, 1996). Cependant, tous les champignons inoculés ne sont pas efficaces et il est encore nécessaire de poursuivre l'effort de recherche avec des objectifs plus étendus en raison de la variabilité des conditions climatiques ou édaphiques dans lesquelles sont établies les plantations d'Eucalyptus en Asie (Aggangan et al., 1996). Nous essayons actuellement de définir des stratégies qui pourraient permettre de mieux intégrer, dans le choix des souches, l'hôte et les facteurs stationnels.

En conclusion, il existe d'importantes possibilités d'amélioration de la croissance des plantations d'Eucalyptus en Asie. Les bénéfices que l'on peut attendre de l'inoculation contrôlée sont largement supérieurs au surcoût généré par la mise en œuvre de cette technique. Nous avons démontré que cette dernière pouvait être appliquée aussi bien dans des pépinières de production à racines nues que dans des pépinières à technologie plus avancée. Néanmoins, pour que le succès de l'inoculation contrôlée soit assuré, encore faut-il prendre bien soin de n'utiliser que des souches compatibles avec l'Eucalyptus et efficaces sur sa croissance. II est indispensable aussi que ces souches aient été au préalable correctement évaluées en conditions réelles de plantation.

\section{B. DELL}

School of Biological and Environmental Sciences MURDOCH UNIVERSITY

PERTH 6150, WESTERN AUSTRALIA

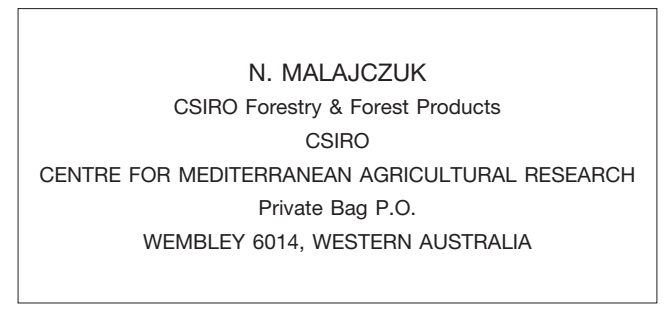




\section{Les possibilités d'application en vue de l'amélioration de la production forestière}

\section{Remerciements}

Les essais du Yunnan ont été mis en place avec l'aide du personnel de l'Academia Sinica (Kunming Institute of Botany). Dans l'île de Mindanao, nous avons bénéficié de l'aide du personnel de la société Bukidnon Forest Industries (Malaybalay), de PICOP (Bislig) et de UPLB Biotech (Los Banos). Nous remercions le Centre international australien pour la Recherche en Agriculture pour leur soutien de longue date à la recherche sur les mycorhizes. Nous remercions Brett Thomson, Tim Grove, Mark Brundrett et Nelly Aggangan pour leur contribution au projet et François Le Tacon pour la traduction de cet article.

\section{BIBLIOGRAPHIE}

AGGANGAN (N.S.), DELL (B.), MALAJCZUK (N.), DE LA CRUZ (R.). - Soil fumigation and phosphorus supply affect the formation of Pisolithus-Eucalyptus urophylla ectomycorrhizas in two acid Philippine soils. - Plant and Soil, vol. 180, 1996, pp. 259-266.

BOUGHER (N.L.). - Diversity of ectomycorrhizal fungi associated with eucalypts in Australia. In: Mycorrhizas for Plantation Forestry in Asia / M. Brundrett, B. Dell, N. Malajczuk, M.Q. Gong Eds. - Canberra : Australian Centre for International Agricultural Research, 1995. - pp. 8-15 (ACIAR Proceedings $\mathrm{n}^{\circ}$ 62).

BRAY (R.H.), KURTZ (L.). - Determination of total, organic, and available forms of phosphorus in soils. - Soil Science, vol. 59,1945 , pp. $39-45$.

BRUNDRETT (M.), BOUGHER (N.), DELL (B.), GROVE (T.), MALAJCZUK (N.). - Working with Mycorrhizas in Forestry and Agriculture. - Canberra : Australian Centre for International Agricultural Research, 1996 (ACIAR Monograph $n^{\circ} 32$ ).

BURGESS (T.I.), MALAJCZUK (N.), GROVE (T.S.). - The ability of 16 ectomycorrhizal fungi to increase growth and phosphorus uptake of Eucalyptus globulus Labill. and E. diversicolor F. Muell. - Plant and Soil, vol. 153, 1993, pp. 155-164.

CASTELLANO (M.A.), BOUGHER (N.L.). - Consideration of the taxonomy and biodiversity of Australian ectomycorrhizal fungi. - Plant and Soil, vol. 159, 1994, pp. 37-46.

DE LA CRUZ (R.E.), BARTOLOME (H.T.), AGGANGAN (N.S.). - Pilot testing of mycorrhizal tablets for pines and Eucalyptus in the Philippines. In : Proceedings UNESCO Regional Workshop on Development and Production of Mycorrhizal Inoculants. - Biotech, UPLB College Laguna, Philippines, 1988.

DE LA CRUZ (R.E.), LORILLA (E.B.), AGGANGAN (N.S.). - Ectomycorrhizal tablets for Eucalyptus species. In : Fast Growing Trees and Nitrogen Fixing Trees / D. Werner, P. Muller Eds. - Stuttgart : Gustav Fischer Verlag, 1990. $-371 \mathrm{p}$.

DELL (B.), MALAJCZUK (N.). - Boron deficiency in eucalypt plantations in China. - Canadian Journal of Forest Research, vol. 24, 1994, pp. 2409-2416.

DELL (B.), MALAJCZUK (N.), GROVE (T.S.). - Nutrient Disorders in Plantation Eucalypts. - Canberra : Australian Centre for International Agricultural Research, 1995 (ACIAR Monograph $n^{\circ} 31$ ).

GARBAYE (J.), DELWAULLE (J.-C.), DIANGANA (D.). - Growth response of eucalypts in the Congo to ectomycorrhizal inoculation. - Forest Ecology and Management, vol. 24, 1988, pp. 151-157.

GROVE (T.S.), LE TACON (F.). - Mycorrhiza in plantation forestry. In : Mycorrhiza Synthesis / I.C. Tommerup Ed. . - Advances in Plant Pathology, vol. 9, 1993, pp. 191-227.

GROVE (T.S.), MALAJCZUK (N.), BURGESS (T.), THOMSON (B.D.), HARDY (G.). - Growth responses of plantation eucalypts to inoculation with selected ectomycorrhizal fungi. In: IUFRO Symposium on Intensive Forestry: The Role of Eucalypts / A.P.G. Schînau Ed. . - Pretoria : South African Institute of Forestry, 1991. - pp. 86-93.

KUEK (C.), TOMMERUP (I.C.), MALAJCZUK (N.). - Hydrogel bead inocula for the production of ectomycorrhizal eucalypts for plantations. - Mycological Research, vol. 96, 1992, pp. 273-277.

MALAJCZUK (N.), BRUNDRETT (M.), DELL (B.). - Concluding remarks and future activities. In: Mycorrhizas for Plantation Forestry in Asia / M. Brundrett, B. Dell, N. Malajczuk, M.Q. Gong Eds. - Canberra : Australian Centre for International Agricultural Research, 1995. - pp. 143-146 (ACIAR Proceedings n 62).

MALAJCZUK (N.), GROVE (T.S.), BOUGHER (N.L.), DELL (B.), GONG MINQIN. - Ectomycorrhizas and nutrients - their importance to eucalypts in China. In: Australian Tree Species Research in China/A.G. Brown. - Canberra: Australian Centre for International Research, 1994. - pp. 132-139.

MALAJCZUK (N.), HARTNEY (V.J.). - Procedures for inoculation of micropropagated plantlets of Eucalyptus camaldulensis with ectomycorrhizal fungi, and comparison with seedling inoculation using inoculum contained in a peat/vermiculite carrier. - Australian Forestry Research, vol. 16, 1986, pp. 199-206.

MARX (D.H.). - The influence of ectotropic mycorrhizal fungi on the resistance of pine roots to pathogenic infections. I. Antagonism of mycorrhizal fungi to root pathogenic fungi and soil bacteria. - Phytopathology, vol. 59, 1969, pp. 153-163.

MARX (D.H.), KENNEY (D.S.). - Production of ectomycorrhizal fungus inoculum. In: Methods and Principles of Mycorrhizal Research / N.C. Schenck Ed. . - Saint-Paul : American Phytopathological Society, 1982. - pp. $131-146$.

MURPHY (J.), RILEY (J.P.). - A modified single solution method for the determination of phosphate in natural waters. - Analitica Chimica Acta, vol. 27, 1962, pp. 31-36. 
THOMPSON (B.D.), GROVE (T.S.), MALAJCZUK (N.), HARDY (G.E. StJ.). - The effectiveness of ectomycorrhizal fungi in increasing the growth of Eucalyptus globulus Labill. in relation to root colonization and hyphal development. - New Phytologist, vol. 126, 1994, pp. 517-524.

THOMPSON (B.D.), HARDY (G.E. StJ.), MALAJCZUK (N.), GROVE (T.S.). - The survival and development of inoculant fungi on the roots of outplanted Eucalyptus globulus Labill. - Plant and Soil, vol. 178, 1996, pp. 247-253.

WALKLEY (A.). - A rapid method for determining organic carbon in soils. - Soil Science, vol. 63, 1947, pp. $251-264$.

\section{L'INOCULATION DES EUCALYPTUS INTRODUITS EN ASIE AVEC DES CHAMPIGNONS ECTOMYCORHIZIENS AUSTRALIENS EN VUE D'AUGMENTER LA PRODUCTIVITÉ DES PLANTATIONS (Résumé)}

La faible fertilité des sols et l'absence de champignons ectomycorhiziens compatibles limitent la croissance des plantations d'Eucalyptus exotiques en Asie. Pendant la dernière décennie, le Centre australien pour la Recherche internationale en Agriculture a soutenu des recherches pour définir les conditions techniques d'inoculation commerciale des plantations d'Eucalyptus. Nous présentons ici plusieurs exemples d'essais de terrain d'inoculation de champignons ectomycorhiziens en sol acide installés en Chine et aux Philippines. Ils concernent une espèce à croissance rapide en climat tempéré (Eucalyptus globulus) et une espèce subtropicale (Eucalyptus urophylla). L'inoculation avec des souches sélectionnées améliore la croissance en hauteur et en surface terrière très rapidement. Trois mois après la plantation, lorsque les jeunes plants n'ont au total que six mois, les effets de l'inoculation sont déjà visibles. Généralement, les différences entre les plants artificiellement inoculés et les témoins augmentent avec le temps. Deux ans après la plantation, le volume de bois produit dans les placeaux inoculés peut être 2,5 fois plus élevé que dans les placeaux témoins. Nous pensons que dans le futur ces résultats entraîneront une intégration des techniques de mycorhization contrôlée dans les pratiques sylvicoles appliquées aux plantations d'Eucalyptus en Asie. Les recherches continuent en Australie pour la sélection de nouvelles souches efficaces dans les conditions les plus difficiles, comme les surfaces couvertes de graminées ou les sols ultramafiques.

\section{INOCULATION OF EUCALYPTS INTRODUCED IN ASIA WITH AUSTRALIAN ECTOMYCORRHIZAL FUNGI FOR THE PURPOSE OF INCREASING PLANTATION PRODUCTIVITY (Abstract)}

Poor soil fertility and lack of compatible ectomycorrhizal fungi limit the extension of exotic eucalypt plantations in Asia. In the past decade, the Australian Center for International Research in Agriculture has sponsored research to define technologies and effective ectomycorrhizal fungi for the commercial inoculation of plantation eucalypts. We present several examples of field trials established in China and the Philippines on acid soils. These involve a fast growing temperate species (Eucalyptus globulus) and a subtropical species (Eucalyptus urophylla). Inoculation with some fungal isolates promoted growth (height, basal area) as early as three months after outplanting, when seedlings were about six months old. Generally, the growth difference between trees inoculated with effective fungi and the uninoculated controls diverged over time. Two years after outplanting, the wood volume produced in inoculated trees was sometimes 2.5 times greater than the controls. We conclude from these trials that ectomycorrhizal inoculation programs will play a significant part in eucalypt sylviculture in Asia in the future. Research is continuing in Australia to target the most suitable eucalyptcompatible ectomycorrhizal fungi for the most difficult sites (i.e., grasslands, ultramafic soils). 